\title{
A Conceptual Approach to Traffic Data Wrangling
}

DOI:

10.1007/978-3-319-60795-5_2

\section{Document Version}

Accepted author manuscript

Link to publication record in Manchester Research Explorer

\section{Citation for published version (APA):}

Al-Jubairah, M., Sampaio, S., Permana, H. A., \& Sampaio, P. (2017). A Conceptual Approach to Traffic Data Wrangling. In A. Cali, P. Wood, N. Martin, \& A. Poulovassilis (Eds.), Data Analytics: Proceedings of 31st British International Conference on Databases, BICOD 2017 (pp. 9-22). (Lecture Notes in Computer Science; Vol. 10365). Springer Nature. https://doi.org/10.1007/978-3-319-60795-5_2

\section{Published in:}

Data Analytics

\section{Citing this paper}

Please note that where the full-text provided on Manchester Research Explorer is the Author Accepted Manuscript or Proof version this may differ from the final Published version. If citing, it is advised that you check and use the publisher's definitive version.

\section{General rights}

Copyright and moral rights for the publications made accessible in the Research Explorer are retained by the authors and/or other copyright owners and it is a condition of accessing publications that users recognise and abide by the legal requirements associated with these rights.

\section{Takedown policy}

If you believe that this document breaches copyright please refer to the University of Manchester's Takedown Procedures [http://man.ac.uk/04Y6Bo] or contact uml.scholarlycommunications@manchester.ac.uk providing relevant details, so we can investigate your claim.

\section{OPEN ACCESS}




\title{
A Conceptual Approach to Traffic Data Wrangling
}

\author{
Mashael Aljubairah ${ }^{1}$, Sandra Sampaio ${ }^{2}$, Hapsoro Adi Permana ${ }^{3}$, and Pedro \\ Sampaio $^{4}$ \\ 1 School of Computer Science, University of Manchester \\ mashael.al-jubairah@postgrad.manchester.ac.uk \\ 2 School of Computer Science, University of Manchester \\ s.sampaio@mancheste r.ac.uk \\ 3 School of Computer Science, University of Manchester \\ hapsoroadi.permana@postgrad.manchester.ac.uk \\ 4 Alliance Manchester Business School, University of Manchester \\ p.sampaio@manchester.ac.uk
}

\begin{abstract}
Data Wrangling (DW) is the subject of growing interest given its potential to improve data quality. DW applies interactive and iterative data profiling, cleaning, transformation, integration and visualization operations to improve the quality of data. Several domain independent DW tools have been developed to tackle data quality issues across domains. Using generic data wrangling tools requires a timeconsuming and costly DW process often involving advanced IT knowledge beyond the skills set of traffic analysts. In this paper, we propose a conceptual approach to data wrangling for traffic data by creating a domain-specific language for specifying traffic data wrangling tasks and an abstract set of wrangling operators that serve as the target conceptual construct for mapping domain-specific wrangling tasks. The conceptual approach discussed in this paper is tool-independent and platform agnostic and can be mapped into specific implementations of DW functions available in existing scripting languages and tools such as R, Python, Trifacta. Our aim is to enable a typical traffic analyst without expert Data Science knowledge to be able to perform basic DW tasks relevant to his domain.
\end{abstract}

Keywords: Data Wrangling, Data Transformation and Quality, Conceptual Wrangling Approaches

\section{Introduction}

Decision makers in different domains, such as healthcare, education and transportation, can gain significant advantages from the enormous volume of available data obtained from various data collection methods, such as site-based sensors, cell-phone tracking [1] and social media. However, data collected via these methods are prone to data quality problems, such as inaccuracy, incompleteness and 
heterogeneity [2] [3]. More recently, data management techniques for data profiling, cleaning and integration have been adapted to improve the quality of large amounts of raw data, in preparation for analysis. The combination of these data management tasks is often called Data Wrangling (DW), generally defined as "the process by which the data required by an application is identified, extracted, cleaned and integrated, to yield a data set that is suitable for exploration and analysis" [4]. According to IBM [5], data analysts spend around 70\% of their time conducting DW activities. Being an interactive and iterative process that involves the application of a variety of data management methods, and that generally lacks a rigid methodology across application domains, DW is often regarded as a highly complex job requiring advanced skills and domain expertise.

Data analysts typically perform DW tasks by using one or a combination of the two following approaches: (i) by programming their own DW applications, using languages such as Python, Java and R; and (ii) by interacting with existing DW tools, which often provide access via a Graphical User Interface (GUI). While approaches (i) and (ii) provide benefits, they also have shortcomings. Approach (i), for example, is often associated with completeness of functionality for fulfilling the requirements of the application in consideration; however, it also involves complex application development, advanced programming skills and brittle solutions that cannot be easily applied over data from other sources than the ones for which the solution was originally designed. On the other hand, approach (ii) is often associated with ease of user interaction, limited need for programming skills, provision of generic functionality that cannot be easily adapted to fulfil specific functional requirements, need for use of multiple tools to perform a single DW job, and limited opportunity for optimization.

To mitigate the limitations identified in approaches (i) and (ii), we propose a conceptual approach and an architectural solution for DW that combines advantages from (i) and (ii), while offering user interaction via a GUI and a high-level and domain-specific declarative language for simple DW tasks. The proposed architecture combines functionality from multiple DW tools and access to multiple data sources, by using Web Services technology. The result is an extensible DW tool, able to take advantage of DW functionality implemented within a variety of existing DW tools, and that provides extensibility and flexibility to allow data analysts to add functionality specific to the requirements of the application in consideration, creating a rich set of DW functions, that can be combined to accomplish simple and complex, general and domain specific DW tasks. For that, high-level user DW requests are automatically mapped into a set of conceptual DW constructs, that are ultimately translated into an execution plan represented as a workflow combining local as well as remote DW functions implemented across a multitude of tools. The proposed approach is tested with use cases from the Urban Traffic Domain, in which DW tasks associated with common data analysis requests by traffic analysts are executed over an implementation of the approach. The paper is organized as follows: Section 2 provides 
a literature review. Section 3 provides the conceptual DW approach including the architecture, conceptual and physical layer design sections, and discussion of implementation aspects. Section 4 provides the conclusions and future work.

\section{Literature Review}

There is a body of research to facilitate DW via Graphical User Interfaces (GUIs) and Domain-Specific Languages (DSLs). For example, in the work of Kandel et al. [6], the complexity of conducting DW was decreased, by associating DW functionality with data visualization constructs, allowing users to conduct DW via a visual interface. Tools offering DW functionality, such as Trifacta [7] and OpenRefine [8], provide concise DSLs combined with GUIs to isolate users from the complexities involved in the wrangling process. Even though both the Trifacta Wrangling Language and the General Refine Expression Language provide data transformation capabilities in Trifacta and OpenRefine, respectively, these languages are not high-level and declarative, and force users to use low-level language constructs. In addition, the level of completeness of functionality provided by these tools varies based on the DW requirements associated with the task at hand, as well as the characteristics of the target data. Considered in isolation, each of these tools will often fail to provide all the operations needed to effectively support the DW capabilities that complex information management problems typically require. A functionality-based comparison for the tools is provided in Tables 3 and 4 in Appendix A.

The difficulties in finding a tool that offers all the functionality required to perform DW tasks forces data analysts to face a steep learning curve before familiarising themselves with multiple tools and experiencing a rather laborious and complex process, in which data often needs to be transformed/reformatted to be transferred between different tools. In addition, data analysts may still have to use low-level programming constructs implemented in languages such as R, Python or Java to be able to customise code and solve specific data quality issues, despite using the DW tools. For example, Bluetooth-based road sensors used to collect traffic data often produce duplicate records for the same moving object due to multiple passengers carrying switched-on Bluetooth devices in a vehicle. For removing duplicates, in this case, multiple attributes need to be considered, such as vehicle identifier, time and location of detection, and device MAC address. However, tools offering DW functionality such as Trifacta Wrangler, OpenRefine and Talend data preparation only provide generic functionality for removing duplicates and so, cannot easily identify non-identical records as duplicates. In addition, because road sensors are generally prone to failure due to environmental conditions and are often able to detect only moving objects equipped with a switched on Bluetooth device, missing data is a common problem in traffic data sets. In the traffic domain, missing data can be replaced with data from the nearest periods or from similar locations or both [9]. Therefore, Spatial Joins using latitude and longitude information are often required 
to address missing data problems and are often not supported by general DW tools. Outliers are also difficult to address in generic DW tools due to the need to include semantic-based information to distingush between outliers and noise. Although there are outlier detection operations distributed across several DW tools, they do not correlate data with other attributes that are important to decide whether the value is an outlier.

\section{A Conceptual Approach to Traffic Data Wrangling}

\subsection{Architectural Overview}

Fig. 1 illustrates the proposed Data Wrangling (DW) architecture. DW requests are expressed in the Declarative Data Wrangling Language $\left(\mathrm{D}^{2} \mathrm{WL}\right)$ and submitted for parsing, during which validation of a $D^{2} W L$ expression is carried out by checking the relevant data sources and other schema information, using meta data. At the end of parsing, a number of expressions in the Data Wrangling Language (DWL) is generated and submitted to optimization. In the current prototype, optimization is based on static information and heuristics, but future work will focus on adaptive and dynamic optimization, based on a cost model. The declarative language is designed for data analysts with limited or no programming skills and so it is based on a small number of clauses that define the location and format of input data sources (the FROM clause), the location and format of results (the TO clause), the main data wrangling activity to be carried out (the WRANGLE clause), and other data wrangling activities using clauses such as GROUPBY. A number of simple functions is also defined within $\mathrm{D}^{2} \mathrm{WL}$ to facilitate the expression of specific data wrangling functions, such as filling in of missing values using function MISSINGDATA(listofattributes) and BY aggregate operation, which defines which data attributes should have their missing values filled in, and how. Fig. 3 provides an example $\mathrm{D}^{2} \mathrm{WL}$ expression that requests attributes gap and headway of a comma-separated values (CSV) file to be filled in by the average value for each of these attributes. Fig. 2 provides the same DW request expressed in English. Note that gap (the time distance between the rear bumper of one vehicle and the front bumper of the vehicle behind it) and headway (the time distance between the front bumpers of two subsequent vehicles) are important traffic congestion indicators, because there is a direct correlation between gap or headway between two vehicles and vehicle speed, and an inverse correlation between the gap or headway and traffic volume. The design of the language is based on an analysis of the most common DW requests by traffic analysts and is able to express a majority of traffic DW requests. The architecture is designed to minimize human interaction by providing automatic translation of $\mathrm{D}^{2} \mathrm{WL}$ expressions into DWL expressions, which are composed of a series of conceptual DW operators needed to perform the requested DW tasks. A GUI will also be provided, via which analysts are able to build complex DW requests, by connecting data sources and abstractions of DW operations as a workflow. Ultimately, each optimized DWL expression is mapped into a number 
of physical DW operations available as services and possibly from different tools and sources.

\subsection{The Conceptual Layer}

The model of data and computation of the proposed Data Wrangling Language (DWL) is functional, with a number of data types and functions, and was designed to be extensible, allowing easy incorporation of additional functions. The data types supported are classified into two main types: atomic and aggregate. Atomic data types include string, character, date, time, boolean and numeric data, such as integer and float. While aggregate data types include general collection types, such as bag, representing an unordered collection of objects allowing duplicates, list, an ordered collection of objects allowing duplicates, set, an unordered collection of objects with no duplicates, and tuple, a record containing objects with different data types. The following symbols are relevant to the DWL example described in Fig. 4: The symbol \lceil\rceil is used to represent an empty bag, $\langle\langle\rangle\rangle$ is used to denote an empty list, [] represents an empty set and \{\} is used to represent an empty tuple. The Collection data type is a generalization of all four collection types, and its constructor is represented by (). An additional data type is Graph, which models visual representations of data.

Fig. 4 describes a DWL expression for the $\mathrm{D}^{2} \mathrm{WL}$ expression in Fig. 3. The read operator takes an URL as input identifying the name, format and location of the input file, possibly on a remote server, and brings the file into context. In this example, this CSV file is described in Appendix A. The enrichTimeStamp operator takes the columnname of a date attribute, and formats it according to the provided format and startdate to add date, month and year information to the attribute. The addColumn operator takes a listofexpressions containing pairs of the type <<columnname, expression>>, describing the names of new columns to be inserted into the file and expressions associated with the column names defining how each new column is to be populated. In this example, the pairs are as follows: <<hour, hour=extractHour (completetimestamp)>, <dayof week, dayof week=extractDayof week (completetimestamp)>>. extract Hour is used to extract the hour from completetimestamp which is the attribute generated by enrichTimeStamp. extractDayof week is similar to extractHour but used to extract the weekday from a date. The result of operator addColumn is the input to selectColumn with a listofcolumnnames, representing the attributes to be projected from the file. The result of selectColumn is input to groupBy, which is also provided with a listofcolumnames that represent the attributes by which records are to be grouped. The fill operator then takes the result of groupBy and replaces the zero values in columns gap and headway with the value resulting from the evaluation of the expression provided in listofexpressions. In the example, the expressions in the list are $<<$ gap=average (gap), headway=average (headway) $>>$, based on the listof conditions which are $\langle<$ if gap $==0$, if headway $==0\rangle>$. Note that the aggregate operations defined in the list of expressions are impacted by how 


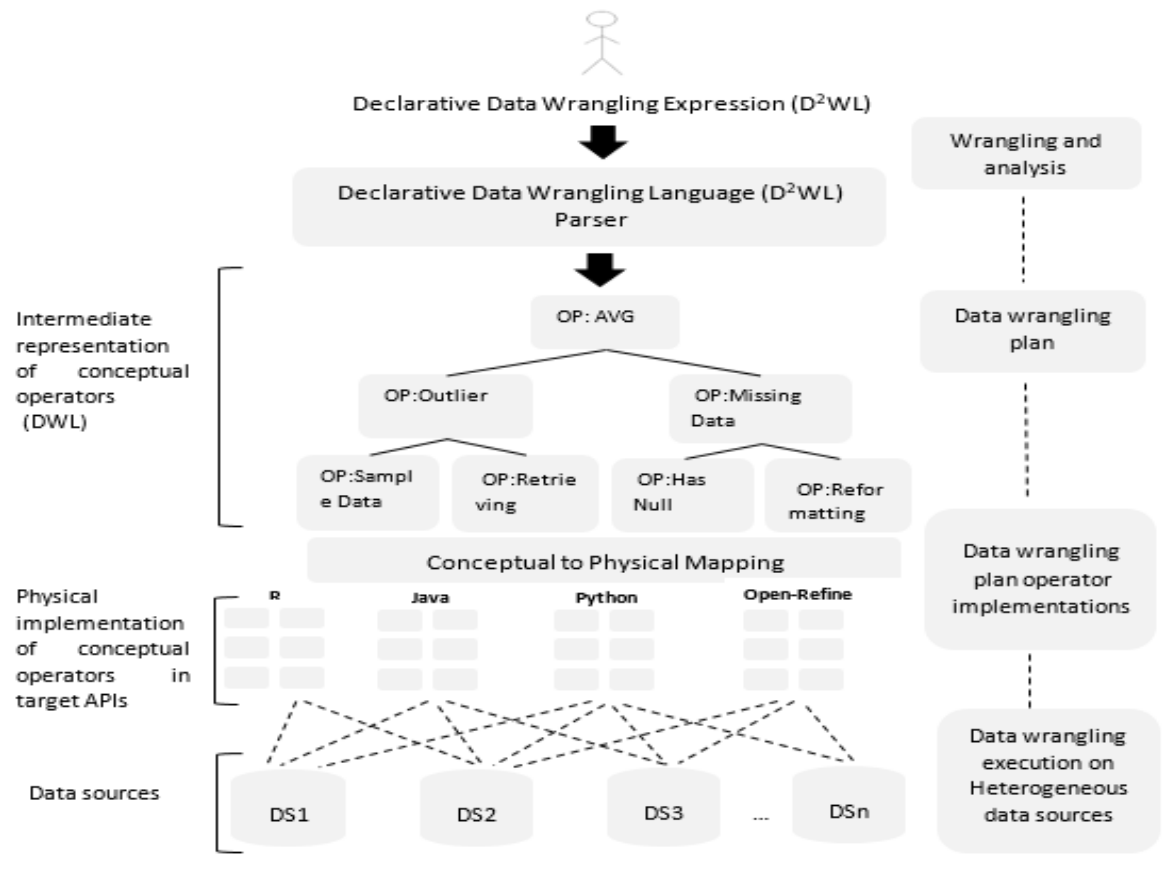

Fig. 1: Data wrangling system architecture.

"Fill in missing values using the average value for headway and gap, and based on observation site, vehicle direction, lane, hour, and day of week, the values for headway and gap could be inferred"

Fig. 2: English expression for the example DW task.

FROM http://www.informationfortraffic.com/Inductive_loops.csv

TO http://www.informationfortraffic.com/Inductive loops.csv

WRANGLE MISSINGDATA(gap, headway)

BY Average

GROUPBY (siteID,vehicleDirection,lane,hour,DayOfWeek)

Fig. 3: $\mathrm{D}^{2} \mathrm{WL}$ expression for the example DW task. 


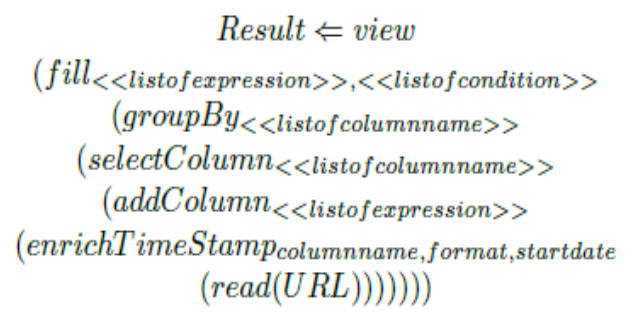

Fig. 4: Conceptual DWL expression for the example DW task.

records are grouped by a groupBy operator. Finally, the view operator allows the user to visualize the result on the screen. A further description of each operation is provided in Appendix B.

\subsection{The Physical Layer}

The Taverna [10] workflow management system is currently being used to call and manage several DW operations implemented within different target tools. The rationale behind the choice of Taverna in the current implementation of the proposed system is its ability to manage complex workflows that require connections to remote services, as well as its ability to allow the annotation and storage of a potentially large number of workflows. Fig. 5 shows an example of how a conceptual DW operation such as read (described in Table 5 of Appendix B) is mapped into a low-level Taverna workflow. Each DW operation is represented as a Taverna workflow, so that the conceptual DW operation name is mapped to a workflow name, the operation argument(s) are mapped into the workflow input port(s) and the operation result(s) are mapped into the workflow output port(s). Each Taverna workflow of a mapped conceptual DW operation includes three processors each of which is associated with a Beanshell or REST service to specify how its functionality is achieved. These services and processors process and pass the input to the workflow via data links which have sink and source variables to identify the connection direction and obtain the target result. Each input and output to a processor is associated with the required input and output to its task-related activity, and the corresponding data link is used to associate the output of the processor with the input to the next processor. Fig. 6 shows the actual execution steps of the Taverna workflow representing the read operation which is implemented as an $\mathrm{R}$ web service. The Beanshell service, EncodeURLfile, is responsible for encoding the input to the REST service, read.csv, implemented in an $\mathrm{R}$ server. This is followed by the Beanshell service, getSessionKey, which obtains the session key associated with the data set brought into context by the read.csv function and makes it available to the next DW conceptual operator, another workflow that will process the data set. 


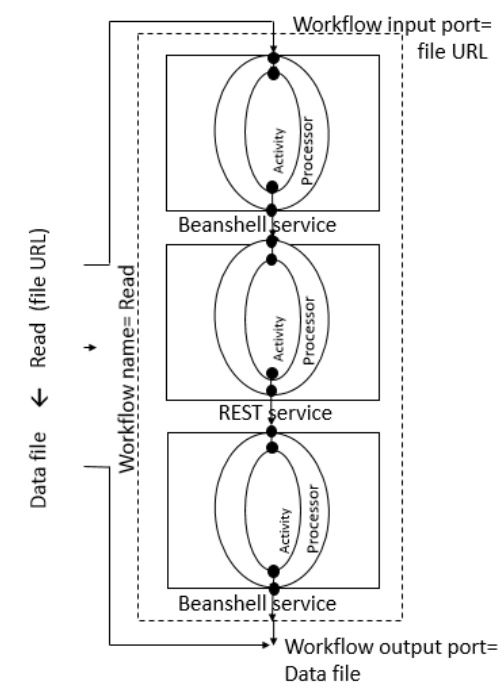

Fig. 5: Details of the Taverna workflow for the conceptual read operation.

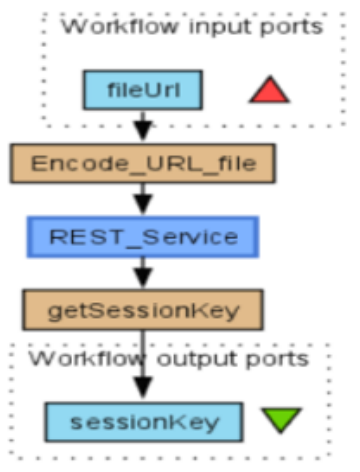

Fig. 6: Execution steps of a Taverna workflow representing the conceptual read operation.

In Section 3.4, further implementation details for the example DW task in Fig. 4 are provided.

\subsection{Implementation of DW operations in Taverna}

Fig. 7 shows the realization of the DW task shown in Fig. 4 as a composition of Taverna workflows. Note that the DWL operators in Fig.4 have been mapped into one or more low-level functions available from two remote servers, one being an R server, and the other one offering DW functionality via simple Python scripts. As described in Section 3.3, Beanshell services have been used to facilitate the HTTP-based communication of data between the various workflows, such as the URL of the file to be input to the task, and a session key associated with an intermediate result that is passed from one workflow to the next. Therefore, each workflow is, in turn, composed of three smaller services, specifically one REST service that encapsulates the main data processing activity associated with the workflow, and two enveloping Beanshell services, the first serving as an input data facilitator and the other one dedicated to extracting the data session key to be output to the next workflow, as Fig. 5 suggests.

In the workflow composition in Fig. 7 the DWL operator, fill, has been mapped into the following calls to $\mathrm{R}$ functions: mutate, to create new columns and impute missing values for gap and headway using expressions and column names as parameters. The expressions include two additional $\mathrm{R}$ operators, replace and average, to replace the missing values using the average value. Two Beanshell services, imputeexpression and colnamesimpute were used to 
provide the mutate parameters values; the groupBy operator has been mapped into R's groupby operator to group the data set based on the provided attributes given by a Beanshell service named colnamesgroupby. Similarly, the selectColumn operator has been mapped into R's selectcolumns to select the required columns provided by colnamesslect Beanshell service; the addColumn operator has been mapped into R's mutate function to create new columns contain information of hour and day of week based on the expressions and column names parameters provided by Beanshell services expressionmutate 2 and colnamesmutate2, respectively. The read operator has been mapped into $\mathrm{R}$ read.csv function to read a remote CSV file, and the enrichTimeStamp has been mapped into completetimestamp Python function to convert the time format from $<$ Minute $><$ Second $><$ Milliseconds $>$ to a more readable format (i.e. 24-hour format) and calculate the date. This operation required begindate, datecolumnname and trafficdataurl as parameters. These parameters are also the workflow input ports as shown in Fig.7. In addition, Taverna allows downloading of the file using the REST service component named getcsv, and the data set was stored into a local CSV file using the Taverna input-output component named writetextfile. The different colours used in Fig.7 indicate different services provided by Taverna. The pink rectangles indicate the encapsulated individual workflows, the brown rectangles indicate Beanshell services, the blue rectangles indicate REST service and the purple rectangle indicates Taverna input-output component.

\section{Conclusions and Future Work}

Due to the complexity associated with the DW process, there is often the need to apply several DW tools, presenting significant challenges to the data analyst. To address these challenges, a novel architectural approach to DW has been proposed. The proposed architecture for DW combines advantages from the existing DW approaches by providing abstract and domain-specific DW constructs without the need for end users to learn low-level programming APIs. In addition, this approach allows data analysts to take advantage of the functionalities available in existing tools. In addition to the Declarative Data Wrangling Language, $\mathrm{D}^{2} \mathrm{WL}$, the conceptual Data Wrangling Language (DWL) can also be complemented by other high-level and declarative domain-specific languages relating to other domains such as education, healthcare and finance. We are currently developing a graphical user interface for traffic analysts to specify $\mathrm{D}^{2} \mathrm{WL}$ expressions and also assistive technology to map from high-level requests to optimised DW strategies implemented in the target platforms. To evaluate the proposed tool, a number of end user experiments will be conducted where analysts from traffic domain are to conduct data wrangling using the tool. A detailed evaluation of the tool will be performed based on both user productivity and tool performance. The user productivity will be measured based on criteria such as learning curve, ease of use, functionality coverage and level of user interaction/effort by quantifying the number of steps required to perform a number of DW tasks of varying com-

plexity. To collect the results, a questionnaire will be provided to data wranglers 


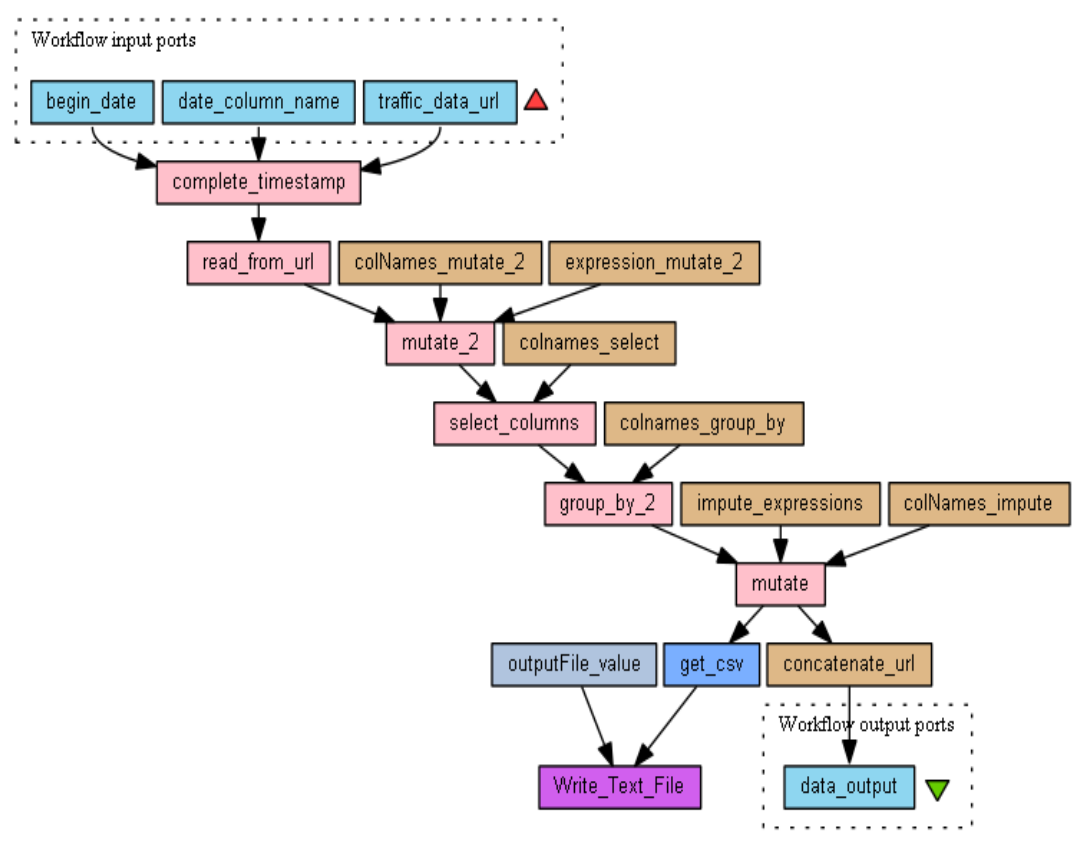

Fig. 7: Implementation of DW task using Taverna.

(i.e. end-users) and performance of existing DW tools will be compared to results obtained from our experiments.

\section{References}

[1] J Lopes, J Bento, E Huang, C Antoniou, and Moshe Ben-Akiva. Traffic and mobility data collection for real-time applications. In Intelligent Transportation Systems (ITSC), 2010 13th International IEEE Conference on, pages 216-223. IEEE, 2010.

[2] Jon Hutchins, Alexander Ihler, and Padhraic Smyth. Probabilistic analysis of a large-scale urban traffic sensor data set. In Knowledge Discovery from Sensor Data, pages 94-114. Springer, 2010.

[3] HV Jagadish, Johannes Gehrke, Alexandros Labrinidis, Yannis Papakonstantinou, Jignesh M Patel, Raghu Ramakrishnan, and Cyrus Shahabi. Big data and its technical challenges. Communications of the ACM, 57(7):86-94, 2014.

[4] Tim Furche, Georg Gottlob, Leonid Libkin, Giorgio Orsi, and Norman W Paton. Data wrangling for big data: Challenges and opportunities. In EDBT, pages 473 478, 2016.

[5] Ignacio Terrizzano, Peter M Schwarz, Mary Roth, and John E Colino. Data wrangling: The challenging yourney from the wild to the lake. In CIDR, 2015.

[6] Sean Kandel, Jeffrey Heer, Catherine Plaisant, Jessie Kennedy, Frank van Ham, Nathalie Henry Riche, Chris Weaver, Bongshin Lee, Dominique Brodbeck, and 
Paolo Buono. Research directions in data wrangling: Visualizations and transformations for usable and credible data. Information Visualization, 10(4):271-288, 2011 .

[7] Trifacta Wrangler Enterprise. Trifacta wrangler. https://www.trifacta.com/, 2015. [Online; accessed 05-01-2016].

[8] open source community Google. Openrefine tool. https://github.com/OpenRefine/OpenRefine/wiki/General-Refine-ExpressionLanguage, 2015. [Online; accessed 28-12-2015].

[9] Chenjuan Guo, Christian S Jensen, and Bin Yang. Towards total traffic awareness. ACM SIGMOD Record, 43(3):18-23, 2014.

[10] Apache Software Foundation. Taverna. https://taverna.incubator.apache.org/[, 2015. [Online; accessed 30-01-2017].

[11] Transport for Great Manchester. Transport for great manchester. http://www.tfgm.com/Pages/default.aspx, 2015. [Online; accessed 17-06-2015].

\section{A Comparison of three widely used data wrangling tools}

This Appendix illustrates the capabilities and limitations of three widely used data wrangling tools in fulfilling a data wrangling task using the traffic data sets depicted in Tables 1 and 2, and illuestrated in Table 3. In addition, it provides a comparion of the three data wrangling tools according to the import and export data format as shown in Table 4. The data wrangling task described as " Retrieve the value of the average speed of vehicles passing road R, (e.g. A572 Manchester Road, Astley Green), on a day of week D (e.g. Monday) at a time interval T (e.g. 8:00 to 9:00)."

\section{B Description of data wrangling operations}

This Appendix provides a description of data wrangling operations as shown in Tables 5,6,7,8,9,10 and 11 .

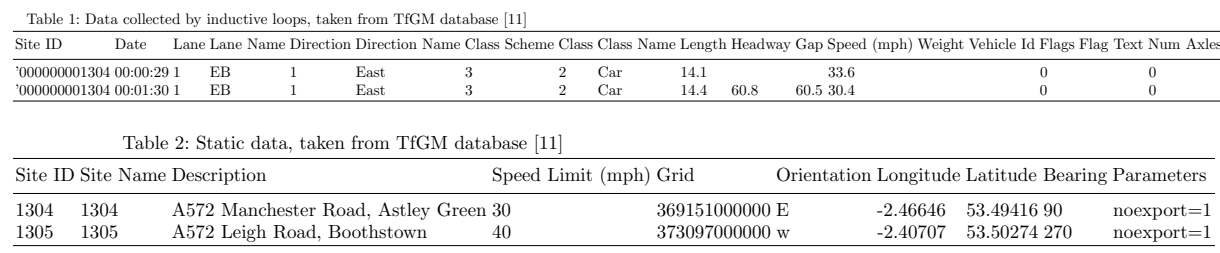


Table 3: Applying the data wrangling task on data wrangling tools

\begin{tabular}{|c|c|c|c|c|}
\hline \multicolumn{2}{|c|}{ Stage No. Description } & \multirow{2}{*}{$\begin{array}{l}\text { OpenRefine } \\
\text { Not supported }\end{array}$} & \multicolumn{2}{|c|}{ Trifacta -free desktop version Talend-data preparation tool } \\
\hline Stage 1 & Check the datasets characteristics:big data & & Supported. & Not supported. \\
\hline Stage 2 & Check the datasets characteristics:CSV data format & Supported. & Supported. & Supported. \\
\hline Stage 3 & Enrich the dataset by Time and Date. & Not supported. & Not supported. & Not supported. \\
\hline Stage 4 & Extract the last four digits of Site ID. & Supported. & Supported. & Supported. \\
\hline Stage 5 & Extract weekday. & Supported. & Supported. & Supported. \\
\hline Stage 6 & Join the data with static data using SiteID as a key. & Supported. & Supported. & Supported. \\
\hline Stage 7 & Filter the data based on weekday, road name and time. & Supported. & Supported. & Supported. \\
\hline Stage 8 & Calculate the average. & Not supported. & Supported. & Not supported. \\
\hline
\end{tabular}

Stage 8 Calculate the average.
Table 4: Data format-based comparison of the data wrangling tools

\begin{tabular}{|c|c|c|c|}
\hline \multirow{2}{*}{$\begin{array}{l}\text { comparison criteria } \\
\text { Input data format }\end{array}$} & \multicolumn{2}{|c|}{ Trifacta -free desktop version OpenRefine } & \multirow[t]{2}{*}{ Talend-data preparation tool } \\
\hline & & & \\
\hline Comma Separated Values (CSV) & Supported. & Supported. & Supported. \\
\hline JavaScript Object Notation(JSON) & Not supported. & Supported. & Not supported. \\
\hline Extensible Markup Language (XML) & Not supported. & Supported. & Not supported. \\
\hline \multicolumn{4}{|c|}{ Export data format } \\
\hline Comma Separated Values (CSV) & Supported. & Supported. & Supported. \\
\hline JavaScript Object Notation(JSON) & Not supported. & Not supported. & Not supported. \\
\hline Extensible Markup Language (XML) & Not supported. & Not supported. & Not supported. \\
\hline
\end{tabular}

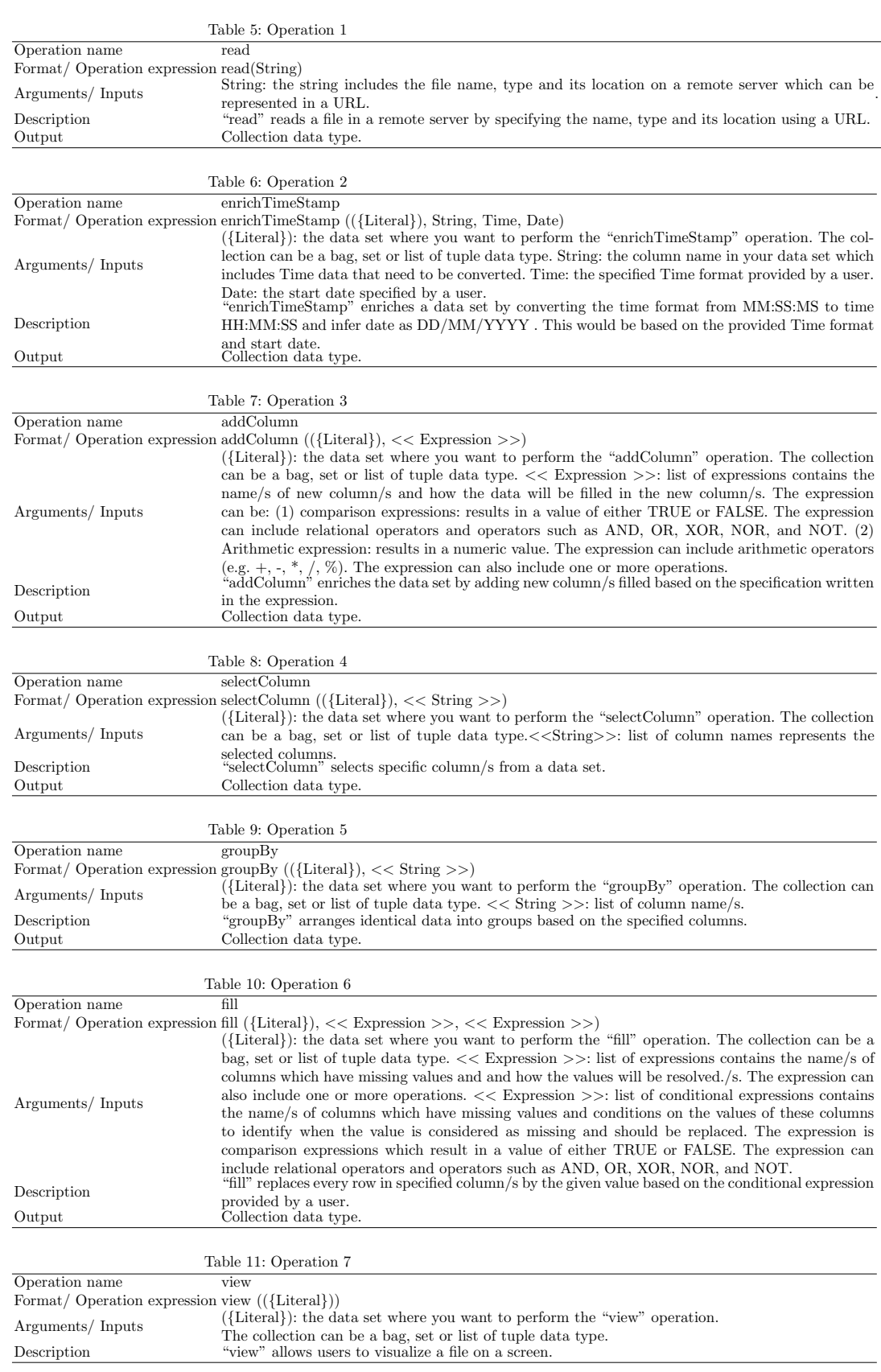

